\title{
Simultaneous Reconstruction of the Activity Image and Registration of the CT Image in TOF-PET
}

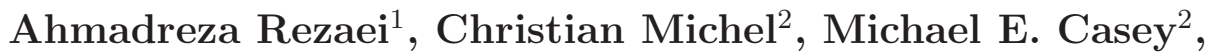 \\ Johan Nuyts ${ }^{1}$ \\ ${ }^{1}$ KU Leuven - University of Leuven, Department of Imaging and Pathology, Nuclear \\ Medicine \& Molecular imaging, Medical Imaging Research Center (MIRC), B-3000 \\ Leuven Belgium \\ ${ }^{2}$ Siemens Medical Solutions, MI, Knoxville, TN 37932, USA. \\ E-mail: ahmadreza.rezaei@uz.kuleuven.be
}

\begin{abstract}
Previously, maximum-likelihood methods have been proposed to jointly estimate the activity image and the attenuation image or the attenuation sinogram from time-of-flight (TOF) positron emission tomography (PET) data. In this contribution, we propose a method that addresses the possible alignment problem of the TOF-PET emission data and the computed tomography (CT) attenuation data, by combining reconstruction and registration. The method, called MLRR, iteratively reconstructs the activity image while registering the available CT-based attenuation image, so that the pair of activity and attenuation images maximise the likelihood of the TOF emission sinogram. The algorithm is slow to converge, but some acceleration could be achieved by using Nesterov's momentum method and by applying a multiresolution scheme for the non-rigid displacement estimation. The latter also helps to avoid local optima, although convergence to the global optimum cannot be guaranteed. The results are evaluated on $2 \mathrm{D}$ and $3 \mathrm{D}$ simulations as well as a respiratory gated clinical scan. Our experiments indicate that the proposed method is able to correct for possible misalignment of the CT-based attenuation image, and is therefore a very promising approach to suppressing attenuation artefacts in clinical PET/CT. When applied to respiratory gated data of a patient scan, it produced deformations that are compatible with breathing motion and which reduced the well known attenuation artefact near the dome of the liver. Since the method makes use of the energy-converted $\mathrm{CT}$ attenuation image, the scale problem of joint reconstruction is automatically solved.
\end{abstract}

Keywords: Time-of-flight positron emission tomography (TOF-PET), joint estimation, maximum likelihood, iterative reconstruction, image registration

\section{Introduction}

Positron emission tomography (PET) data are acquired over a relatively long time interval whereas computed tomography $(\mathrm{CT})$ attenuation values are acquired almost instantaneously. In addition, in current PET/CT scanners, the CT and PET scans 
are acquired sequentially. Thus, artifacts due to patient and/or breathing motion are expected in emission reconstructions (in-scan motion) together with artifacts due to possible misalignment of PET and CT data (between-scan motion). Recent studies have shown that in time-of-flight (TOF) PET, joint and stable estimation of the activity and the attenuation is possible (Defrise et al. 2012; Salomon et al. 2011; Rezaei et al. 2012b). Given the availability of TOF-PET data, the activity image can be jointly estimated either with the attenuation image (Salomon et al. 2011; Rezaei et al. 2012b; Rezaei et al. 2012a) or with the attenuation sinogram (Rezaei et al. 2014; Defrise et al. 2014; Panin et al. 2012; Li et al. 2013). Because TOF-PET determines the attenuation only up to a constant (Defrise et al. 2012), some constraining is required for accurate quantitative reconstruction. The MLAA algorithm (Rezaei et al. 2012b; Boellaard et al. 2014) jointly estimates the two images, solving for the constant by imposing the known attenuation of tissue. Alternatively, Mehranian et al. (2015b) solved the scale problem by incorporating an intensity prior on the estimated attenuation values using a Gaussian mixture model of different tissue types. For MLACF (Rezaei et al. 2014; Defrise et al. 2014), which jointly estimates the activity image and the attenuation sinogram, this straightforward constraining method is not possible. However, Panin et al. (2012) proposed a modified MLACF version to complete the attenuation factors obtained from $\mathrm{CT}$, in which the constant is determined by the available CT-data.

Although the use of consistency conditions for the estimation of attenuation from non-TOF emission data (Natterer et al. 1992; Kudo et al. 2001; Bronnikov 2000) has had limited success in practice, they were found to be useful in determining the strength of regularisation parameters for maximum a-posteriori reconstruction of the attenuation image from transmission measurements (Panin et al. 2004). Furthermore, studies have shown that the consistency conditions of the non-TOF emission data can also be used to estimate the attenuation image that has affected the measurements as an affine transform of a known attenuation image (Natterer 1993; Welch et al. 1998). This approach was used as a means to correct for attenuation in the case of between-scan motion (Bromiley et al. 2001; Alessio et al. 2010). Although instability issues were reported with an affine motion model (Bromiley et al. 2001), the results seemed to be encouraging with a rigid motion model despite a slow convergence (Alessio et al. 2010). However, the problem of correcting for patient and/or breathing motion requires more complex transformation models. Since with the introduction of TOF, the PET emission data provide more information about the attenuation than before, it is expected that the TOF-PET data also provide a means to estimate more complex motion models, and could help mitigate the problems of in-scan and between-scan motion in TOF-PET.

In current TOF-PET/CT systems, the PET attenuation image is normally estimated by converting the CT-image values to the photon energy of $511 \mathrm{keV}$ (Kinahan et al. 2003). In this study, we use this CT-derived attenuation image in a joint estimation framework and align the attenuation image so that the pair of the activity image and the deformed CT-based attenuation image better explain the emission measurements. To do so, the estimation of the attenuation image of MLAA (Rezaei et al. 2012b) is replaced 
with a transformation (rigid or non-rigid) estimation based on minimising a sum of squared differences (SSD) term in the joint estimation framework. We show below that weighing the SSD term by the curvature of the emission log-likelihood ensures that a decrease of the SSD term will produce an increase of the quadratic surrogate function for the likelihood. The proposed algorithm is called MLRR (maximum likelihood reconstruction of activity and registration of attenuation), which aims at combining the high signal to noise ratio of the $\mathrm{CT}$ image with the optimal (i.e. the maximum likelihood) alignment produced by the joint estimation. Since the method makes use of CT-based attenuation images, no extra correction is required for the missing scale problem in the joint estimation method.

The paper is organised as follows; the MLRR algorithm is described in section 2. We will also look at options to improve its convergence, since the method was shown to have a slow convergence rate with a non-rigid motion model (Rezaei et al. 2013a). The design of the $2 \mathrm{D}$ and $3 \mathrm{D}$ simulation experiments are presented in section 3 . The simulation results are shown together with the results of a respiratory gated clinical scan in section 4. In section 5, we conclude by discussing the results, drawing some conclusions and giving an outline of future research on the topic.

\section{Method/Theory}

Assuming Poisson statistics and ignoring constant terms, the log-likelihood function for TOF-PET emission data $y$ can be expressed as:

$$
L(\lambda, \mu, y)=\sum_{i t} y_{i t} \ln \bar{y}_{i t}-\bar{y}_{i t}
$$

where $\lambda$ and $\mu$ represent the emission and attenuation parameters and $\bar{y}_{i t}$ is the expected emission sinogram value for line-of-response (LOR) $i$ and TOF-bin $t$, which is computed as:

$$
\bar{y}_{i t}=b_{i t} a_{i}+s_{i t}=\sum_{j} c_{i j t} \lambda_{j} e^{-\sum_{k} l_{i k} \mu_{k}}+s_{i t}
$$

where $b_{i t}$ is the TOF-projection of the activity image along LOR $i$ and in TOF-bin $t$, $a_{i}$ is the attenuation factor along the same LOR and $s_{i t}$ represents the contributions of scatter and/or randoms for the same data bin $i, t$. Furthermore, $c_{i j t}$ represents the sensitivity of detector $i$ and TOF-bin $t$ to emissions coming from voxel $j, \lambda_{j}$ is the activity in voxel $j, l_{i j}$ is the effective intersection length between LOR $i$ and voxel $j, \mu_{j}$ is the attenuation at voxel $j$. Note that summation over the TOF index $(t)$ yields the corresponding non-TOF values $\left(\sum_{t} c_{i j t}=c_{i j}\right.$ and $\left.\sum_{t} y_{i t}=y_{i}\right)$.

The MLRR algorithm treats the log-likelihood function as a function of the activity $\lambda$ and the attenuation $\mu[\Theta]$, where $\Theta=\left\{\Theta_{p} \mid p=1 \ldots P\right\}$ represents a set of deformation parameters, which deform the known attenuation image $\mu^{\dagger}$ into the attenuation image $\mu[\Theta]$. The log-likelihood is maximised by estimating the activity values $\lambda_{j}$ in every voxel $j$ and the deformation parameters $\Theta$. Below, an iterative algorithm is derived which 
alternately updates the activity values while keeping the attenuation image fixed, and then updates the deformation parameters while keeping the activity values fixed.

When the deformation, and therefore the attenuation, is fixed, the problem of updating the activity image is the same as in standard maximum likelihood expectation maximisation (MLEM) reconstruction. Hence, the activity is updated by applying an iteration of the MLEM algorithm or its accelerated version, ordered subsets expectation maximisation (OSEM). When the activity is fixed, the deformation of the attenuation map must be updated such as to increase the likelihood. This problem could be solved by deriving a dedicated gradient ascent algorithm for this subproblem. Instead, we propose to use a nested approach, which first computes a desired attenuation update using an established ML algorithm for transmission tomography (MLTR (Van Slambrouck et al. 2014)) and then apply an established registration algorithm to find the (incremental) deformation that results in a good approximation of that desired update. In section 2.1 this method is derived, essentially by applying the chain rule to the gradient of the likelihood and introducing reasonable approximations. This leads to the algorithm (12) - (14). In section 2.2 an acceleration scheme based on Nesterov's momentum method is proposed. We consider both rigid and non-rigid deformations of the attenuation map. For rigid deformations, the second step of the nested approach (eq. (14)) is solved with a weighted least squares registration algorithm, as briefly discussed in section 2.3. Section 2.4 proposes a method for non-rigid deformations, where the deformation step is computed with a slightly modified version of the demons algorithm (Thirion 1998).

\subsection{Attenuation Deformation Estimation}

When the activity is fixed, the log-likelihood function becomes similar to that of standard transmission tomography, except that instead of updating the attenuation values, we wish to adjust the attenuation image by modifying the deformation parameters.

The proposed algorithm is based on the MLTR algorithm (Van Slambrouck et al. 2014), a maximum likelihood reconstruction algorithm for transmission tomography. In every iteration MLTR makes a quadratic approximation to the Poisson likelihood function, which is then replaced by a separable quadratic surrogate function. The surrogate function has the following form:

$$
S\left(\mu^{(n)}+\delta \mu, y\right)=L\left(\mu^{(n)}, y\right)+\sum_{j} \dot{L}_{j}^{(n)} \delta \mu_{j}+\sum_{j} \frac{1}{2} \ddot{L}_{j}^{(n)}\left(\delta \mu_{j}\right)^{2}
$$

where,

$$
\left.\dot{L}_{j}^{(n)} \triangleq \dot{L}_{j}\right|_{\mu=\mu^{(n)}}=\left.\frac{\partial L}{\partial \mu_{j}}\right|_{\mu=\mu^{(n)}},\left.\quad \ddot{L}_{j}^{(n)} \triangleq \ddot{L}_{j}\right|_{\mu=\mu^{(n)}}=\left.\sum_{k} \frac{\partial^{2} L}{\partial \mu_{j} \partial \mu_{k}}\right|_{\mu=\mu^{(n)}}
$$

where $\mu^{(n)}$ represents the attenuation reconstruction at the current iteration $n$ and $\delta \mu$ 
is its update. Maximising (3) produces the MLTR update $\delta \mu^{(n)}$ :

$$
\begin{aligned}
& \mu_{j}^{(n+1)}=\mu_{j}^{(n)}+\delta \mu_{j}^{(n)} \\
& \delta \mu_{j}^{(n)}=\underset{\delta \mu}{\arg \max } S\left(\mu^{(n)}+\delta \mu, y\right)=-\frac{\dot{L}_{j}^{(n)}}{\ddot{L}_{j}^{(n)}}
\end{aligned}
$$

Details on the MLTR update for TOF-PET data are given in Appendix A. The update maximises the surrogate and guarantees that the value of the quadratic approximation to the likelihood increases. The likelihood is therefore expected to increase as well, but because of the approximation, monotonicity cannot be guaranteed. In our experience this algorithm works very well in practice and increases the likelihood monotonously.

The MLTR algorithm is now adapted to the new problem using an approach similar to that proposed by Wang and Qi (Wang et al. 2009) for direct reconstruction of kinetic parameters. For the problem at hand, the surrogate function (3) is rewritten as a function of the deformation parameters $\Theta$ :

$$
\tilde{S}\left(\mu\left[\Theta^{(n)}\right]+\delta \mu[\theta], y\right)=L\left(\mu\left[\Theta^{(n)}\right], y\right)+\sum_{j} \dot{L}_{j}^{(n)} \delta \mu_{j}[\theta]+\sum_{j} \frac{1}{2} \ddot{L}_{j}^{(n)}\left(\delta \mu_{j}[\theta]\right)^{2}
$$

where $\Theta^{(n)}$ is the deformation at the current iteration $n, \theta$ is an update to that deformation and $\delta \mu[\theta]=\mu\left[\Theta^{(n)}+\theta\right]-\mu\left[\Theta^{(n)}\right]$. The new value of $\Theta$ is obtained as

$$
\begin{aligned}
& \Theta^{(n+1)}=\Theta^{(n)}+\theta^{(n)} \\
& \theta^{(n)}=\underset{\theta}{\arg \max } \tilde{S}\left(\mu\left[\Theta^{(n)}\right]+\delta \mu[\theta], y\right)
\end{aligned}
$$

where we have slightly misused the ' + ' sign to denote the composition of the transformations. The update is obtained by setting the derivatives of (6) to zero, which yields:

$$
\frac{\partial \tilde{S}}{\partial \theta_{p}}=\left(\sum_{j} \dot{L}_{j}^{(n)}+\ddot{L}_{j}^{(n)} \delta \mu_{j}[\theta]\right) \frac{\partial \delta \mu_{j}[\theta]}{\partial \theta_{p}}=0, \quad p=1, \ldots, P
$$

This maximisation is equivalent to the following least squares problem:

$$
\theta^{(n)}=\underset{\theta}{\arg \min } \frac{1}{2} \sum_{j} \ddot{L}_{j}^{(n)}\left(\delta \mu_{j}[\theta]-\delta \mu_{j}^{(n)}\right)^{2}
$$

Finally, (10) can be regarded as an image registration problem using a weighted least squares criterion, since it can be rewritten as:

$$
\theta^{(n)}=\underset{\theta}{\arg \min } \frac{1}{2} \sum_{j} \ddot{L}_{j}^{(n)}\left(\left(\mu_{j}\left[\Theta^{(n)}\right]+\delta \mu_{j}[\theta]\right)-\left(\mu_{j}\left[\Theta^{(n)}\right]+\delta \mu_{j}^{(n)}\right)\right)^{2}
$$

The two images that are registered are

- the static image $\mu\left[\Theta^{(n)}\right]+\delta \mu^{(n)}$, which is obtained by applying MLTR iteration (5) to the image obtained in the current iteration, and

- the image $\mu\left[\Theta^{(n)}\right]+\delta \mu[\theta]$, which is obtained by deforming the image at the current iteration according to the deformation parameters $\theta$. 
Consequently, the MLRR algorithm is obtained by iterating the following three steps procedure:

(i) apply MLEM to update $\lambda_{j}$ :

$$
\lambda_{j}^{(n+1)}=\frac{\lambda_{j}^{(n)}}{\sum_{i} c_{i j} a_{i}} \sum_{i t} c_{i j t} a_{i} \frac{y_{i t}}{\sum_{\xi} c_{i \xi t} a_{i} \lambda_{\xi}^{(n)}+s_{i t}}
$$

(ii) apply the MLTR algorithm to obtain the intermediate reconstruction $m^{(n)}$ (see Appendix A for expressions for $\dot{L}_{j}$ and $\ddot{L}_{j}$ ):

$$
m_{j}^{(n)}=\mu_{j}\left[\Theta^{(n)}\right]+\delta \mu_{j}^{(n)}=\mu_{j}\left[\Theta^{(n)}\right]-\dot{L}_{j}^{(n)} / \ddot{L}_{j}^{(n)}
$$

(iii) apply a weighted least squares registration to update the deformation:

$$
\Theta^{(n+1)}=\Theta^{(n)}+\underset{\theta}{\arg \min } \frac{1}{2} \sum_{j} \ddot{L}_{j}^{(n)}\left(\mu_{j}\left[\Theta^{(n)}\right]+\delta \mu_{j}[\theta]-m_{j}^{(n)}\right)^{2}
$$

\subsection{Acceleration}

We have observed previously that the MLRR algorithm is slow to converge (Rezaei et al. 2012b). Here we introduce a term based on Nesterov's momentum (Kim et al. 2014; Nesterov 1983) that affects the estimation of the intermediate reconstruction $m^{(n)}$, and significantly improves the convergence speed of the algorithm. In this accelerated scheme, the second step of the algorithm (eq. (13)) is replaced by:

(ii)* replace MLTR update $m^{(n)}$ with the update $m^{(n)^{*}}$, which is obtained with Nesterov's momentum method as follows:

$$
\begin{aligned}
m_{j}^{(n)^{*}} & =\mu_{j}\left[\Theta^{(n)}\right]+\delta \mu_{j}^{(n)^{*}} \\
& =\mu_{j}\left[\Theta^{(n)}\right]-\left.\frac{\dot{L}_{j}}{\ddot{L}_{j}}\right|_{\mu=\mu\left[\Theta^{(n)}\right]+\alpha^{(n)} \delta \mu^{(n-1)^{*}}}+\alpha^{(n)} \delta \mu_{j}^{(n-1)^{*}}
\end{aligned}
$$

where, $\delta \mu^{(n)^{*}}$ is the current accelerated MLTR attenuation update, and

$$
\alpha^{(n)}=\frac{h^{(n-1)}-1}{h^{(n)}}, \quad h^{(n)}=\frac{1+\sqrt{1+4\left(h^{(n-1)}\right)^{2}}}{2}
$$

with $h^{(0)}=1$.

The addition of a term based on previous MLTR reconstruction updates $\delta \mu^{(n-1)^{*}}$ provides some momentum for attenuation reconstruction, and consequently for the estimated motion parameters. The acceleration with Nesterov momentum is known to be non-monotonic even if the original optimiser is monotonic.

\subsection{Special Case of Rigid Motion Estimation}

To simultaneously reconstruct the activity image and rigidly align the attenuation map, the parameter set $\Theta$ contains 6 rigid motion parameters (three translations and three rotation angles). They are determined by a rigid registration algorithm which minimises the sum of weighted squared differences (11), using a gradient descent algorithm and accelerated by incorporating the momentum produced by (15). 


\subsection{Special Case of Non-rigid Deformation Estimation}

For the non-rigid case, the deformation parameters $\Theta$ are replaced by a vector $\ddagger$ $\boldsymbol{D}_{j}=\left(D_{j}^{x}, D_{j}^{y}, D_{j}^{z}\right)$ of displacement fields for every voxel $j$. Minimising (10) by means of the demons registration algorithm (Thirion 1998), an incremental displacement $\boldsymbol{d}$ update is computed as:

$$
\boldsymbol{d}_{j}^{(n)}=\frac{\left(\boldsymbol{\nabla} \mu\left[\boldsymbol{D}^{(n)}\right]\right)_{j} \delta \mu_{j}^{(n)}}{\left\|\left(\boldsymbol{\nabla} \mu\left[\boldsymbol{D}^{(n)}\right]\right)_{j}\right\|^{2}-\beta / \ddot{L}_{j}^{(n)}}
$$

where $\left(\nabla \mu\left[\boldsymbol{D}^{(n)}\right]\right)_{j}$ is the gradient of the deformed attenuation image at voxel $j$ with respect to the image coordinates $(x, y, z)$, and $\beta / \ddot{L}_{j}^{(n)}$ helps to stabilise the incremental displacement estimate $\boldsymbol{d}^{(n)}$ at low gradient values of the deformed attenuation image in which $\beta$ is the strength of a penalty term. Details on the derivation of the incremental displacement update are given in Appendix B.

The displacement update (17) is essentially the constrained demons update (Thirion 1998), differing from it only by using a different form for the stabilisation term and by only using the gradient of the deformed CT image§. The original demons algorithm uses $\|\delta \mu\|^{2}$ in the denominator which automatically limits the step size to a maximum of 0.5 pixels. As the iterations proceed and the maximum likelihood solution is approached, the values $\delta \mu$ become small, hence a smaller maximum step size would be more desirable. The use of the stabilising term $\beta / \ddot{L}_{j}^{(n)}$ further reduces the influence of the vanishing $\delta \mu_{j}^{(n)}$ in later iterations. Unfortunately $\ddot{L}_{j}^{(n)}$ is count-dependent. This count-dependency is accounted for by adjusting $\beta$ such that the incremental displacement update of (17) gives a maximum change of 0.5 voxels in the early iterations of the MLRR algorithm. The value of $\beta$ is then kept constant in subsequent iterations, ensuring that the displacement updates (17) will vanish with vanishing $\delta \mu_{j}$.

When using the acceleration provided by the momentum of the attenuation updates (using the accelerated estimate $\delta \mu^{(n)^{*}}$ defined in (15) instead of $\delta \mu^{(n)}$ ), in order to limit the maximum incremental displacement in (17) to the defined maximum, the same acceleration scheme must also be applied to the stabilising term $\beta / \ddot{L}^{(n)}$. Since $\beta$ is fixed and $\ddot{L}^{(n)}$ changes little with iterations, we alternatively weight the strength of the regularisation term in each iteration by:

$$
\gamma^{(n)}=1+\alpha^{(n)} \gamma^{(n-1)}
$$

where, $\gamma^{(0)}=0$ and use $\gamma^{(n)} \beta / \ddot{L}_{j}^{(n)}$ as the modified regularisation term in (17).

The task of image registration can also be accelerated by a multi-resolution registration scheme. A multi-resolution approach offers two advantages: not only it accelerates the registration process, it also reduces the risk of getting stuck at possible local maxima of the solution space. However, with a non-rigid motion model, convergence to the global solution is never guaranteed. In our implementation of the

$\ddagger$ A bold symbol indicates a three element vector throughout the text.

$\S$ The gradient of the moving image is used as opposed to the gradient of the fixed image (Vercauteren et al. 2009). 
multi-resolution registration scheme, the incremental displacement update is estimated in multiple resolution levels in every iteration, where the pixel width in a coarser level was set to twice that of the finer resolution level. After updating the incremental displacement fields in each resolution level, the final incremental displacement field is computed as the composition of the multi-resolution incremental displacement fields (coarse-to-fine resolution levels), and is used to deform the CT attenuation image.

In addition to constraining the incremental displacement update to small displacement values by introducing a quadratic penalty term defined in (B.5), we also favour locally smooth displacement estimates $\boldsymbol{d}_{j}$ by enforcing a fluid-like and a diffusionlike smoothing on the estimated displacement fields. These approaches have been previously shown to be very effective for optimisation with the demons (Vercauteren et al. 2009). When computing the non-rigid displacement fields at each resolution level, the additional regularisation was done by applying a Gaussian smoothing to the displacement fields, i.e.:

$$
\begin{aligned}
& \boldsymbol{d}_{j}^{(n)}=\sum_{\xi} G_{j \xi}^{F} \boldsymbol{d}_{\xi}^{(n)} \\
& \boldsymbol{D}_{j}^{(n+1)}=\sum_{\xi} G_{j \xi}^{D}\left(\boldsymbol{D}_{\xi}^{(n)}+\boldsymbol{d}_{\xi}^{(n)}\right)
\end{aligned}
$$

where, $G^{F}$ and $G^{D}$ are the fluid-like and diffusion-like regularising Gaussian kernels (Vercauteren et al. 2009; Cachier et al. 2004), respectively. Details about the parameter values used in the experiments are provided in the simulation designs of section 3.

When the activity is fixed and the log-likelihood is treated as a function of voxel attenuation values, the associated Hessian is negative semi-definite, and therefore, the only local maximum is the global one (Lange et al. 1995). However, when the loglikelihood is treated as a function of a displacement field, the computation of the Hessian is challenging, and it seems very likely that there will be multiple local maxima. Therefore, starting the non-rigid motion estimation from a rigidly aligned attenuation map would be recommended.

\section{Experiment Design}

The Siemens Biograph mCT scanner specifications (Jakoby et al. 2011) (with a radial detector mashing of 2) were chosen in the simulations. In the $2 \mathrm{D}$ simulations, the TOF-PET data were organised in a 3D sinogram consisting of 200 radial bins of 0.4 cm width, 168 projection angles over $180 \mathrm{deg}$, and 13 TOF-bins of 312 ps width (which is sufficient to avoid aliasing artifacts in the reconstructions (Defrise et al. 2008)) with an effective TOF resolution of 580 ps. A small discrepancy between the simulation and reconstruction projectors was introduced by discretising the $2 \mathrm{D}$ thorax phantom in an over-sampled grid of $600 \times 600$, and the simulated LORs were subsequently undersampled as the average of three LORs. Both activity and attenuation images were forward-projected using Joseph's method (Joseph 1982), where for each TOF-bin the 
activity image was weighted by the effective weights of the TOF-bin width and the TOFresolution. Activity and attenuation images were then reconstructed in a $200 \times 200$ pixel grid of $0.4 \mathrm{~cm}$ width.

In the fully 3D simulation, the TOF-PET emission data were organised in a 5D sinogram consisting of 200 radial bins of $0.4 \mathrm{~cm}$ width, 168 azimuthal angles, 9 copolar angles with 109 planes of $0.2 \mathrm{~cm}$ width, and 13 TOF-bins of 312 ps width. A 3D implementation of the above forward-projection was used to obtain the 5D TOF-PET emission measurements. The resulting $3 \mathrm{D}$ activity and attenuation reconstructions had a $200 \times 200 \times 109$ voxel grid with a voxel width of $0.4 \mathrm{~cm}$ and $0.2 \mathrm{~cm}$ in the transaxial and axial directions, respectively.

The above described MLRR images were compared to the reconstructions obtained with the MLAA algorithm (Rezaei et al. 2012b), with the aim of revealing similarities and differences between the joint reconstructions and not identifying one as the algorithms of choice (left for future research). The MLAA reconstructions were initialised with a uniform activity image and a uniform tissue attenuation image in the phantom/patient support. In order to eliminate any confounding effects of the scale factor in the reconstructions, the scale problem in MLAA was fixed by assuming knowledge of the total tracer activity. The MLRR algorithm is initialised with a uniform activity image, the misaligned CT-based attenuation image $\mu^{\dagger}$ and the identity displacement field for a non-rigid motion model. Furthermore, the CT attenuation image used in the simulations had the same image resolution as the true phantoms that were used to create the PET emission data. In the patient data reconstructions, a Gaussian smoothing of $4 \mathrm{~mm}$ FWHM was applied to correct for the difference in PET and CT system resolutions.

In the following, the activity and attenuation figures are displayed in inverse gray and gray colour maps, respectively.

\subsection{D Simulation}

The MLRR and MLAA algorithms were compared in a 2D TOF-PET simulation of a $2 \mathrm{D}$ thorax phantom. Figure 1 shows the activity and attenuation images with three (tissue (blue), lung (green), and tumour (red)) contours of the regions of interest (ROIs) where the reconstructions are analysed in. The figure also shows the two mismatched CT attenuation images used in the study. The mismatch in the CT1 attenuation image is created by an increase of the size of the lungs, and a change in the size and location of the simulated tumour lesion. The CT2 attenuation image is obtained by a rigid transformation of the CT1 attenuation image, i.e. rotation of $30 \mathrm{deg}$, and a translation of $2.4 \mathrm{~cm}$ and $6.0 \mathrm{~cm}$ in the horizontal and vertical directions, respectively.

3.1.1. Reconstructions MLAA and MLRR reconstructions are compared for noisefree TOF-PET data as well as moderate-noise and high-noise in the emission data. The moderate-noise TOF-PET data had an expected maximum count of 50.4 and 146.0 


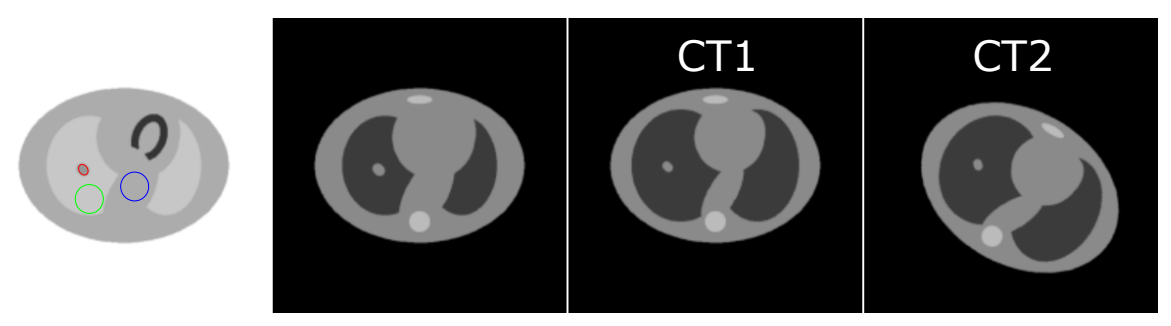

Figure 1: 2D activity (column 1) and attenuation (column 2) images of a simulated thorax phantom. The activity contours define tissue (blue), lung (green), and tumour (red) regions of interest (ROIs). The two mismatched CT attenuation images used in the 2D simulation study are shown in columns 3 and 4. CT1 (column 3) differs from the true attenuation by a non-rigid deformation of the attenuation image, CT2 (column 4 ) is obtained by a rigid transformation of the CT1 attenuation image.

in the TOF-PET sinogram and its corresponding non-TOF sinogram data bins, for the high-noise data these count values were 12.6 and 36.5. In this study, the CT2 attenuation image was used as the mismatched attenuation image. For MLAA, we assumed that the total amount of activity was known and the MLAA activity reconstructions were scaled in each iteration accordingly.

The reconstructions are analysed after 5 iterations of 24 subsets of the MLRR algorithm, where the attenuation image is updated three times for each update of the activity image (i.e. after each MLEM sub-iteration, three MLTR sub-iterations are applied). The first iteration estimates the rigid transformation parameters, providing an initial alignment of the given CT attenuation image. The following 4 iterations assume a non-rigid deformation model, where the multi-resolution scheme is used to update the displacement parameters in two resolution levels in each iteration. A diffusionlike and fluid-like regularisation of the displacement field was obtained by smoothing the displacement field and its update with a Gaussian of 1.0 and 2.5 pixels FWHM, respectively. The same smoothing regularisation was applied for the noise-free and the noisy TOF-PET emission data sets. For the MLAA reconstructions, 3 iterations with 24 subsets were applied, again with 3 MLTR sub-iterations for each MLEM subiteration. For the MLEM reconstruction 3 iterations with 24 subsets were computed. In both MLAA and MLRR, the attenuation is updated more frequently than the activity because its convergence tends to be slower.

The emission data provide no attenuation information in LORs that do not intersect the activity distribution. In order to improve the attenuation reconstruction of MLAA, LOR-values outside the support of the activity distribution were given a small count in the "blank" (un-attenuated projection of the activity image) and measured sinograms, which encourages the MLTR algorithm to assign zero attenuation outside the activity distribution support. For MLRR, the displacement estimation was restricted to the voxels within the support of the CT-based attenuation image. 
3.1.2. Bias-Variance We analysed noise properties of the activity reconstructions of both MLRR and MLAA. First a noise-free data set was computed (with the same expected maximum count as the high-noise data set of section 3.1.1), which was then used as the expectation for a pseudo-random Poisson noise generator to generate 100 independent noise realisations. In this study, the CT2 mismatched attenuation image was used, and the reconstruction parameters were set as previously described. The results are reported after 120 updates of MLRR and MLAA activity reconstructions, where all projection angles were used in every update. For MLRR, a rigid motion model is used in the first 24 activity updates and a non-rigid motion model is used for the following 96 updates of the MLRR activity image. The joint activity reconstructions are compared to MLEM reconstructions with the attenuation sinograms of the true, CT1, and CT2 attenuation images. We assume that the noise-free reconstructions of the thorax phantom are a good estimate of the average of noisy reconstructions. Bias is computed as the average absolute pixel-by-pixel difference between the noise-free reconstruction and the activity phantom of figure 1 , and variance is computed as the squared mean difference from the noise-free reconstructions. We report the estimated bias as well as the variance of activity reconstructions in the support of the $2 \mathrm{D}$ thorax phantom for 100 different TOF-PET noise realisations.

3.1.3. Convergence Analysis In order to get more insight into the convergence properties of MLRR, the log-likelihood of equation (1) as well as the root mean square error (RMSE) between the attenuation reconstructions and the true attenuation image were computed for each iteration of MLRR. The log-likelihood was normalised by its upper limit defined by the TOF emission data, i.e. $\sum_{i t} y_{i t} \ln y_{i t}-y_{i t}$. The influence of Nesterov's momentum acceleration as well as the multi-resolution displacement estimation scheme in MLRR are analysed in the noise-free and the moderate-noise TOF-PET emission data. A non-rigid motion model starting from the CT1 mismatched attenuation image was used in the analysis, and the likelihood and RMSE are plotted for 250 updates of the attenuation image. In this study, no ordered subsets acceleration was applied, i.e. all projection angles were used in every iteration.

3.1.4. TOF-resolution The effects of the TOF-resolution was studied by varying the simulated TOF Gaussian kernel ranging from 1.2 ns to 0.2 ns FWHM. In order to avoid aliasing artifacts, the emission data were projected in 40 TOF-bins of 100 ps width. Similar to section 3.1.3, the algorithm is initialised with the CT1 mismatched attenuation image, only a non-rigid motion model was considered and no ordered subsets were applied during reconstructions. We report on the likelihood and RMSE obtained after 250 updates of the attenuation image. 


\subsection{D Simulation}

The XCAT phantom (Segars et al. 2010) was used to generate realistic respiratory motion. A maximum diaphragm motion of $2.0 \mathrm{~cm}$ and a maximum anterior-posterior motion of $1.2 \mathrm{~cm}$ was used to simulate the breathing cycle, which was gated into 8 (motion-free) frames. Figure 2 shows the activity and attenuation images of the XCAT phantom frame used in this study as well as the mismatched CT attenuation image which was chosen from a different frame in the respiratory cycle.
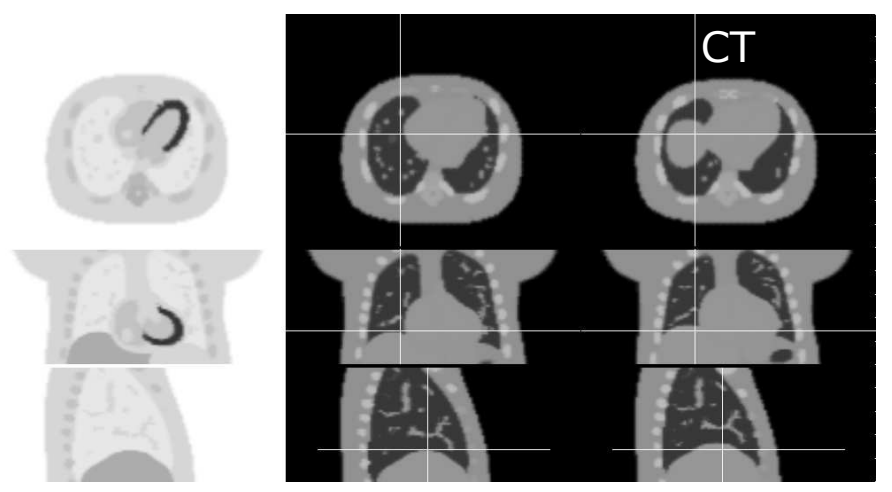

Figure 2: Transaxial, coronal and sagittal views through the true activity (left), true attenuation (centre), and the mismatched CT attenuation (right) images of the breathing XCAT phantom. The XCAT phantom was gated into 8 respiratory gates, the true activity and attenuation images, and the mismatched attenuation image correspond to frames 4 and 2 , respectively.

The XCAT phantom was forward projected with the 3D TOF-PET projector, and Poisson noise was added to the measurements to simulate a 4 min ${ }^{18} \mathrm{~F}-\mathrm{FDG}$ thorax scan. The average maximum count of the TOF sinogram and its corresponding non-TOF sinogram were 10.6 and 28.2, respectively. MLRR and MLAA activity and attenuation reconstructions are then compared. Furthermore, the attenuation reconstruction of MLRR is compared to a demons (Thirion 1998) registration of the CT attenuation image and the reference true attenuation of figure 2 . In the $3 \mathrm{D}$ simulation, the displacement estimate of MLRR was regularised by a diffusion-like smoothing of 1.0 voxel FWHM and a fluid-like smoothing of 2.5 voxels FWHM. The activity and attenuation reconstruction comparison of MLAA and MLRR are after 3 iterations of 24 subsets, and the demons registered attenuation image is obtained after the same number of registration updates in two resolution levels.

\subsection{Patient Data}

3.3.1. Reconstruction A clinical 4 min TOF-PET thorax scan (Siemens Biograph $\mathrm{mCT}$ ) of a patient injected with $296 \mathrm{MBq}$ of ${ }^{18} \mathrm{~F}-\mathrm{FDG}$ is reconstructed with MLRR and compared to activity reconstructions of MLEM. The emission data was acquired 80 minutes post-injection, and had a measured true-to-prompt coincidence event ratio 
of $36 \%$. The additive contribution of randoms as well as the single-scatter estimate (Watson 2007) were corrected for during reconstructions. The displacement estimates were updated as in section 3.2 .

3.3.2. Gated Reconstructions The amplitude-based data-driven gating of (van Elmpt et al. 2011) is used to gate the $4 \mathrm{~min}{ }^{18} \mathrm{~F}$-FDG thorax scan of the patient into 3 respiratory gates. For each gate, we compare the MLEM activity reconstruction with the CT-based attenuation image to the activity reconstruction of MLRR, initialised with the same CT-based attenuation image.

\section{Results}

\subsection{D Simulation}

4.1.1. Reconstructions An MLEM activity reconstruction with the mismatched CT2 attenuation image is shown in figure 3 together with the joint activity and attenuation reconstructions of MLAA and MLRR for the noise-free TOF-PET emission data. Figure 3 also shows the attenuation estimates of MLRR (after 1 iteration of 24 subsets) assuming a rigid transformation model and the non-rigid attenuation estimate of MLRR (the following 4 iterations of 24 subsets). The MLRR attenuation estimate is comparable to the attenuation reconstruction of MLAA; however, it differs slightly near rapid directional changes of the attenuation gradient.

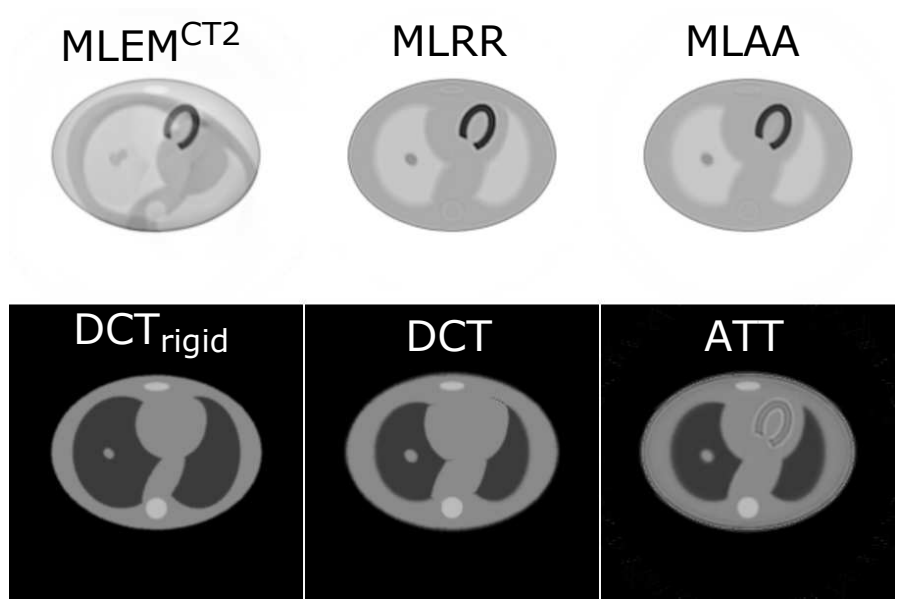

Figure 3: The top row shows the activity images produced by MLEM with the mismatched CT2 attenuation image, by MLRR and by MLAA. The bottom row shows the MLRR attenuation images obtained after the initial rigid transformation (left) and the final non-rigid transformation (middle), and the MLAA attenuation image (right). (The mismatched CT2 attenuation image is shown in fig 1.)

Figure 4 shows the MLRR and MLAA activity and attenuation reconstructions for the moderate-noise and the high-noise TOF-PET emission data. As in the noisefree case, the MLRR attenuation reconstructions are obtained after an initial rigid 
parameter estimation followed by a non-rigid displacement estimation. Since noise behaves differently in both algorithms, the attenuation reconstructions are visually different. In MLAA noise directly influences the voxel values whereas in MLRR noise propagates through the deformation into the position of the anatomical boundaries (i.e. gradients in the attenuation image), however, the resulting attenuation image is still piecewise smooth similar to the original CT-based attenuation image. Thus, implicitly applying a very strong noise suppression to the attenuation coefficients, except near anatomical boundaries.
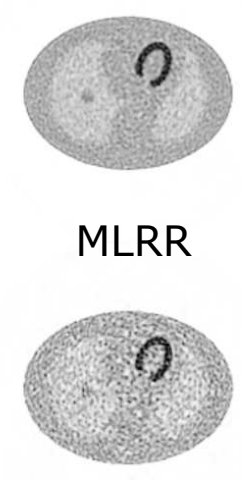

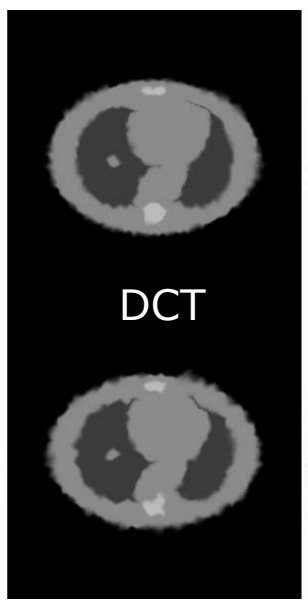

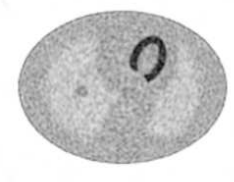

MLAA

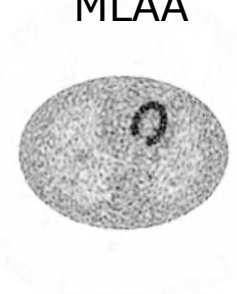

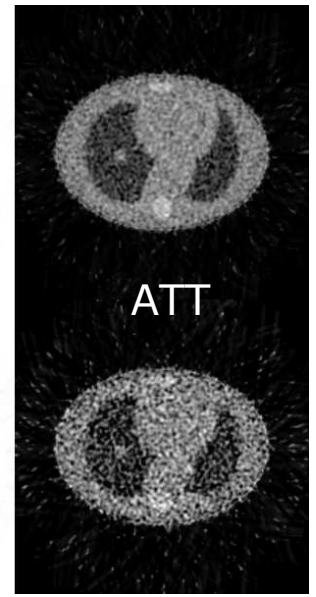

Figure 4: Activity (columns 1 and 3) and attenuation (columns 2 and 4) reconstructions of MLRR and MLAA for the moderate-noise (top) and high-noise (bottom) TOF-PET emission data.

At both noise levels, the activity reconstructions of MLAA and MLRR are very similar. To quantify the accuracy of the reconstructions, two error measures were used; 1- the mean absolute difference (MAD) and 2- the mean difference (MD) of the reconstructions. The error terms were computed as:

$$
\mathrm{MAD}=\frac{\sum_{j}\left|\mathrm{ACT}_{j}-\mathrm{GT}_{j}\right|}{\sum_{j} \mathrm{GT}_{j}}, \quad \mathrm{MD}=\frac{\sum_{j \in \mathrm{ROI}} \mathrm{ACT}_{j}-\sum_{j \in \mathrm{ROI}} \mathrm{GT}_{j}}{\sum_{j \in \mathrm{ROI}} \mathrm{GT}_{j}}
$$

where GT is the ground truth activity distribution (figure 1) and ACT was set to the activity reconstruction of MLRR, MLAA and MLEM ${ }^{\mathrm{CT} 2}$, respectively. For the reconstructions of figure 4, the mean absolute difference of MLRR and MLAA were $28.9 \%$ and $26.5 \%$ for the moderate-noise activity reconstructions and $51.1 \%$ and $48.8 \%$ for the high-noise simulations, respectively. The same measure of error was $42.8 \%$ and $50.6 \%$ for the MLEM reconstruction with the mismatched CT attenuation image. Table 1 reports on the mean difference (MD) errors obtained in the ROIs defined in figure 1. Although the MLRR and MLAA produce similar error terms in the defined ROIs, the results could still be affected by a difference in convergence of the two methods.

4.1.2. Bias-Variance Figure 5a shows the bias and variance properties of MLRR and MLAA activity reconstructions compared to reference MLEM reconstructions with the 
Table 1: Mean difference (MD) errors of different regions of interest (ROIs) for MLRR/MLAA activity reconstructions and the ground truth activity of figure 1.

\begin{tabular}{l|cc|cc|cc|} 
& \multicolumn{2}{c}{ Tumor ROI } & \multicolumn{2}{c}{ Tissue ROI } & \multicolumn{2}{c}{ Lung ROI } \\
\cline { 7 - 8 } & MLRR & MLAA & MLRR & MLAA & MLRR & MLAA \\
\hline noise-free (\%) & 7.0 & 2.9 & 2.6 & 0.6 & 1.9 & -0.7 \\
moderate-noise (\%) & -4.0 & -1.6 & -6.9 & -4.8 & 2.4 & -3.9 \\
high-noise (\%) & -3.0 & -10.1 & -6.1 & -7.5 & -0.8 & -0.3
\end{tabular}

true $\left(\mathrm{MLEM}^{\text {True }}\right)$, CT1 (MLEM $\left.{ }^{\mathrm{CT} 1}\right)$, and CT2 (MLEM $\left.{ }^{\mathrm{CT} 2}\right)$ attenuation images. As expected, the misalignment of the CT can strongly influence bias in the emission reconstructions. Interestingly, after changing from a rigid motion model (prior to the kink in the curve) to a non-rigid motion model, bias and variance values for MLRR and MLAA are similar. Figure 5a suggests that similar bias should be expected by both MLRR and MLAA at a matched variance level. Furthermore, figure 5b shows the scatter plots of the MLRR/MLAA and the MLEM mean and variance activity reconstructions of the 100 noise realisations, where linear regression was applied to quantify image similarities. For both the mean and the variance images, slightly better similarity measures (slope $q$, coefficient of determination $r^{2}$ ) were computed for MLRR than for MLAA compared to the reference MLEM mean and variance images.

4.1.3. Convergence Analysis Figure 6 shows the normalised likelihood (1) and the RMSE values for 250 updates of the MLRR algorithm where no subsets were used for the estimation of the MLTR attenuation update. The two measures are computed with no acceleration and a registration in only the finest resolution level (MLRR). The results are then compared to the results obtained by using Nesterov's momentum $\left(\mathrm{MLRR}^{+}\right)$, registration in two resolution levels in each iteration $\left(\mathrm{MLRR}^{\mathrm{MR}}\right)$, and the combined acceleration and multi-resolution scheme $\left(\mathrm{MLRR}^{\mathrm{MR}+}\right)$. Although monotonicity of the likelihood cannot be guaranteed in MLRR, the likelihood has increased in each iteration in all simulated cases. In all simulations, the reconstructions accelerated by Nesterov's momentum achieve a higher likelihood and a lower RMSE than the nonaccelerated MLRR reconstructions. Furthermore, the joint utilisation of Nesterov's momentum together with multi-resolution registration scheme provides an increased level of convergence with the least amount of error. It should also be mentioned that applying ordered subsets lead to further acceleration of the algorithm; however, the decrease in the RMSE values was no longer monotonic.

4.1.4. TOF-Resolution Figure 7 shows the estimated likelihood and the RMSE of the reconstructed activity images compared to the ground truth activity image of figure 1. It can be observed that as the TOF-resolution improves so does the convergence of the MLRR algorithm. Analysing the RMSE for different sets of fluid-like and diffusion-like regularisation of the estimated displacements, we found that the fluid-like regularisation 


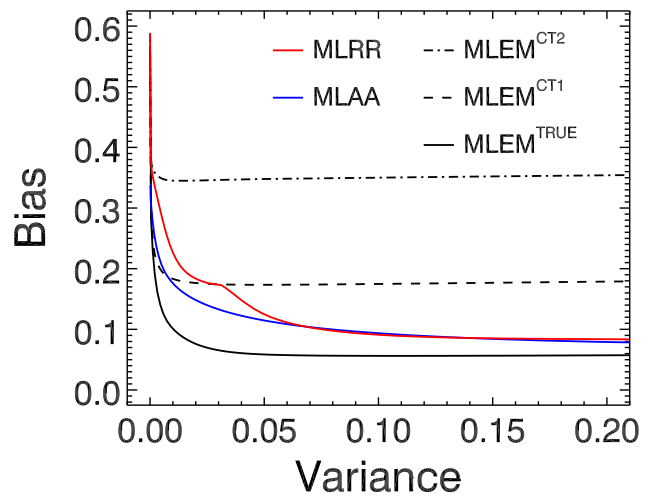

(a) Bias and variance of MLRR and MLAA activity reconstructions compared to MLEM activity reconstructions with the true, CT1, and CT2 attenuation images. The curves are produced by varying the number of iterations.
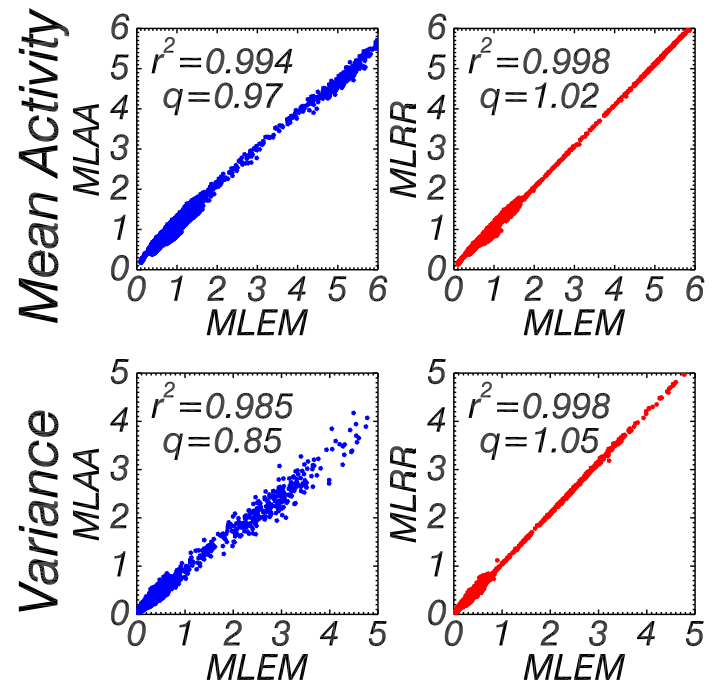

(b) MLAA-MLEM (left) and MLRR-MLEM (right) scatter plots of the mean (top) and the variance (bottom) of the activity reconstructions (obtained after 120 updates) for the 100 noise realisations. Linear regression (slope $q$, coefficient of determination $r^{2}$ ) was applied to the data-points of each plot to analyse the image similarities.

Figure 5: Results of the noise realisation study, (a): bias and variance curves, (b): MLRR and MLAA scatter plots.

had a minimal effect on the RMSE values, whereas the diffusion-like regularisation could cause instabilities most likely occurring at discontinues of the gradient image of the attenuation.

\subsection{D Simulation}

Figure 8 shows the MLRR and MLAA activity reconstructions as well as the MLEM activity reconstructions with the true attenuation image (MLEM ${ }^{\text {True}}$ ) and the mismatched CT (MLEM ${ }^{\mathrm{CT}}$ ) image which was chosen from a different respiratory frame. As expected, the MLEM reconstruction suffers from motion artifacts (most pronounced near the dome of the liver), and the joint activity reconstructions of MLAA and MLRR are comparable to the MLEM reconstruction with the true attenuation image (MLEM $\left.{ }^{\text {True }}\right)$. The figure also shows the attenuation reconstruction of MLAA (ATT) and the attenuation estimate of MLRR (DCT) together with a registered demons attenuation image. Although the attenuation reconstruction of MLAA is sufficient for attenuation correction, the attenuation details are lost due to the amount of noise in the data. In contrast, the MLRR attenuation estimate has the benefit of producing an aligned attenuation estimate while roughly maintaining the resolution of the mismatched CT attenuation image. Visual inspection shows a good agreement between MLRR 

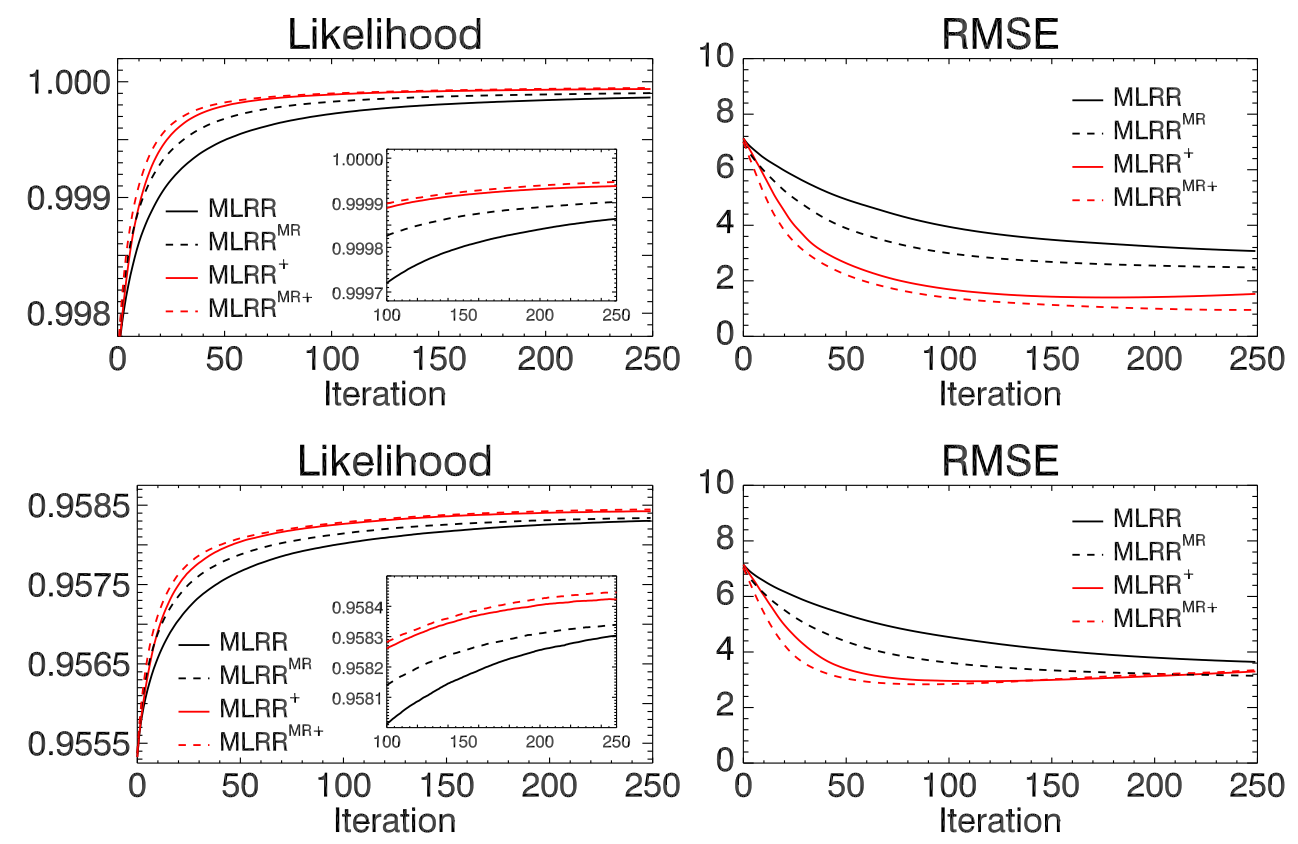

Figure 6: Normalised log-likelihood of (1) (left) together with the RMSE (right) of the attenuation estimates of MLRR for noise-free (top) and moderate-noise (bottom) TOF-PET emission data.
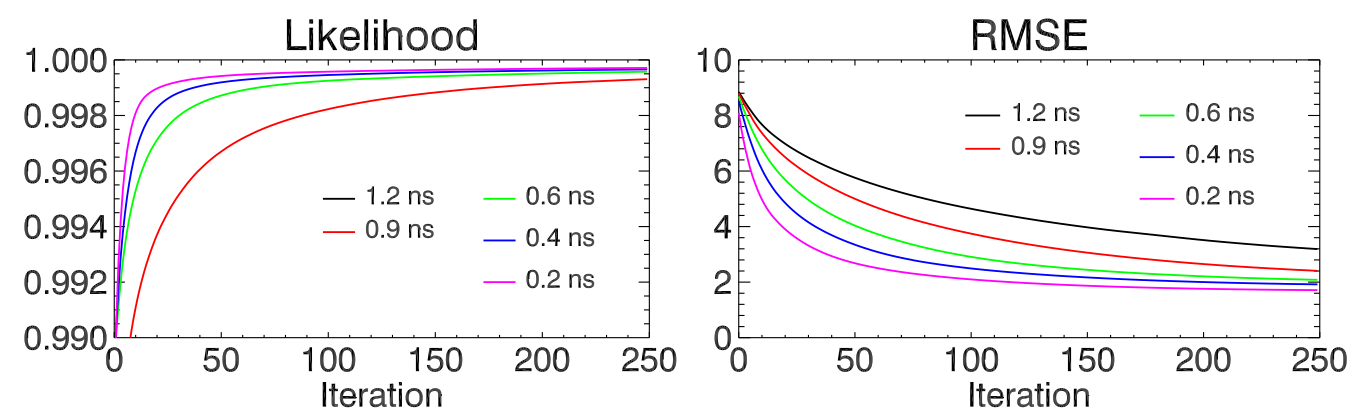

Figure 7: Normalised log-likelihood of (1) (left) together with the RMSE (right) of the activity reconstructions of MLRR for the noise-free TOF-PET emission data with a varying TOF Gaussian resolution of 1.2 ns to 0.2 ns FWHM.

attenuation image and the true attenuation image.

\subsection{Patient Data}

4.3.1. Reconstruction Figure 9 shows the MLEM activity reconstruction together with the MLRR and MLAA activity and attenuation reconstructions of the $4 \mathrm{~min}{ }^{18} \mathrm{~F}-\mathrm{FDG}$ patient scan. The reconstructions are obtained after 3 iterations of 24 subsets, and the activity reconstructions are post-smoothed by a Gaussian of $0.4 \mathrm{~cm}$ FWHM. The shadow-like artifacts (more pronounced near the dome of the liver and the lateral wall of the heart) in the MLEM activity reconstruction suggest slight mismatch between the CT and the average attenuation image that has affected the TOF-PET emission 


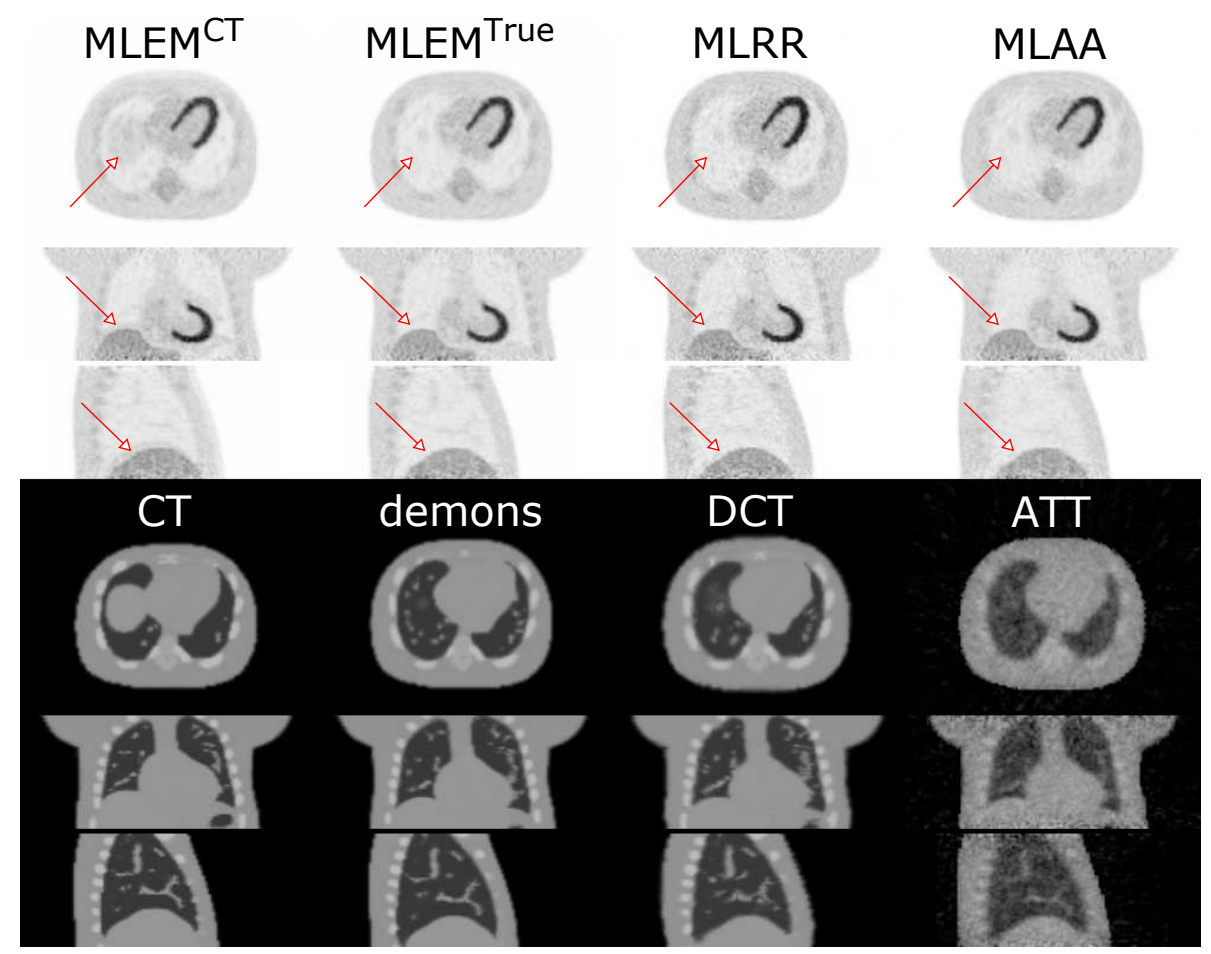

Figure 8: Activity (top) and attenuation (bottom) reconstructions of the XCAT phantom. The MLRR (column 3) and MLAA (column 4) activity reconstructions are compared to activity reconstructions of MLEM with the mismatched CT (column 1 top) and the true attenuation image (column 2 - top). The CT (column 1 - bottom) and the registered demons (column 2 - bottom) attenuation images are shown as reference. For the true attenuation image, see figure 2 (centre column).

data. The reconstructed attenuation image of MLRR (and MLAA) supports this claim, where the boundary of the liver is shifted upward and hence the activity reconstruction of MLRR (and MLAA) seems free of the shadow-like artifacts. It has been shown that TOF-PET is more robust than non-TOF PET to errors in the attenuation image (Conti 2011). Hence, to better reveal the presence of possible attenuation mismatches, nonTOF data were produced by summing all TOF-bins and reconstructed with non-TOF MLEM using the different attenuation maps. The comparison of these non-TOF MLEM images based on the CT, the deformed CT of MLRR (DCT) and the MLAA (ATT) attenuation images provide more evidence that the alignment of attenuation image has been improved by the joint estimation methods.

4.3.2. Gated Reconstructions Figure 10 shows the MLRR reconstruction of the same 4 min ${ }^{18}$ F-FDG patient scan when the emission data was gated into 3 respiratory gates. As before, the reconstructions are obtained after 3 iterations of 24 subsets and the activity reconstructions are post-smoothed by a $0.4 \mathrm{~cm}$ FWHM Gaussian kernel. It is interesting to see that the position of the lung-liver boundary differs for the attenuation reconstruction of each gate, and that the MLRR activity reconstructions seem to be free 


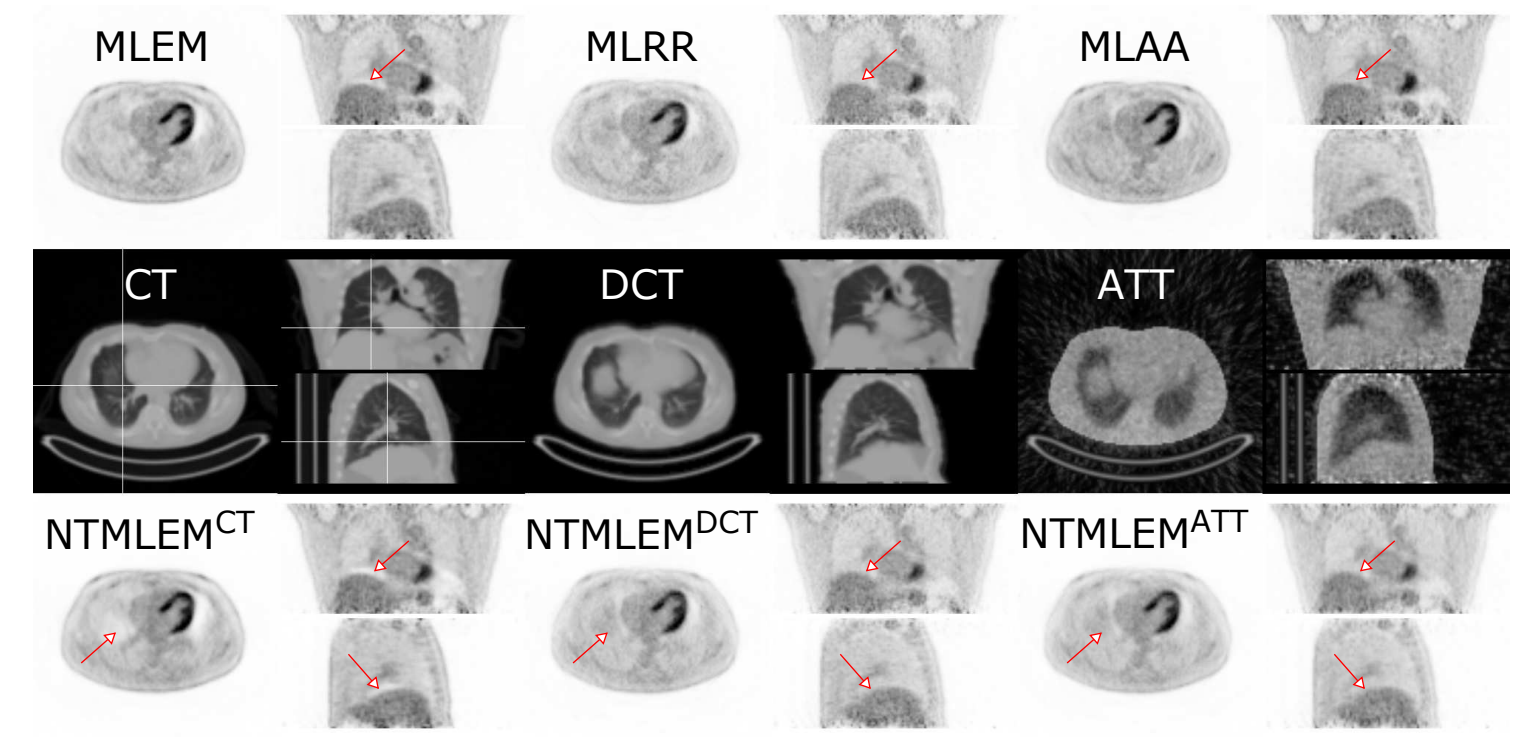

Figure 9: Transaxial, coronal and sagittal slices through the activity (top) reconstructions of MLEM (left), MLRR (centre) and MLAA (right) shown together with the CT-based attenuation (middle-left), the MLRR deformed-CT attenuation (middlecentre) and the MLAA attenuation (middle-right) reconstruction. The non-TOF (intensifying motion-induced artifacts) MLEM activity reconstructions (bottom) with the CT-based (left), MLRR deformed-CT (centre) and the MLAA (right) attenuations are shown for reference.

of the shadow-like artifacts observed in the MLEM activity reconstruction close to the boundary of the liver. Although the shadow-like artifacts near the lateral wall of the heart seem to be reduced in the MLRR activity reconstruction, there are some remaining artifacts which are observed in both MLRR and MLAA activity reconstructions which we attribute to the cardiac motion.

\section{Discussion}

Time-of-flight PET has attracted a lot of interest among researchers due to the desired properties of the emission reconstructions from TOF-PET data (Conti 2011), e.g. faster convergence rate, improved contrast-to-noise ratio, robustness to possible inaccuracies in normalisation and attenuation factors, etc. More recently a systematic study on a population of clinical patient scans (Mehranian et al. 2015a) has showed that TOF reconstructions substantially improve the quantitative accuracy of tracer distribution as a result of the TOF robustness to possible imperfections. Since it was shown that the TOF-PET emission data provide information about the attenuation that is not available in non-TOF data (Defrise et al. 2012; Conti 2011; Salomon et al. 2011), the topic of joint activity and attenuation reconstruction from TOF-PET data has gained increased attention. In addition to the desired properties of TOF-PET reconstructions, the joint reconstruction methods provide a novel approach to activity and attenuation alignment 


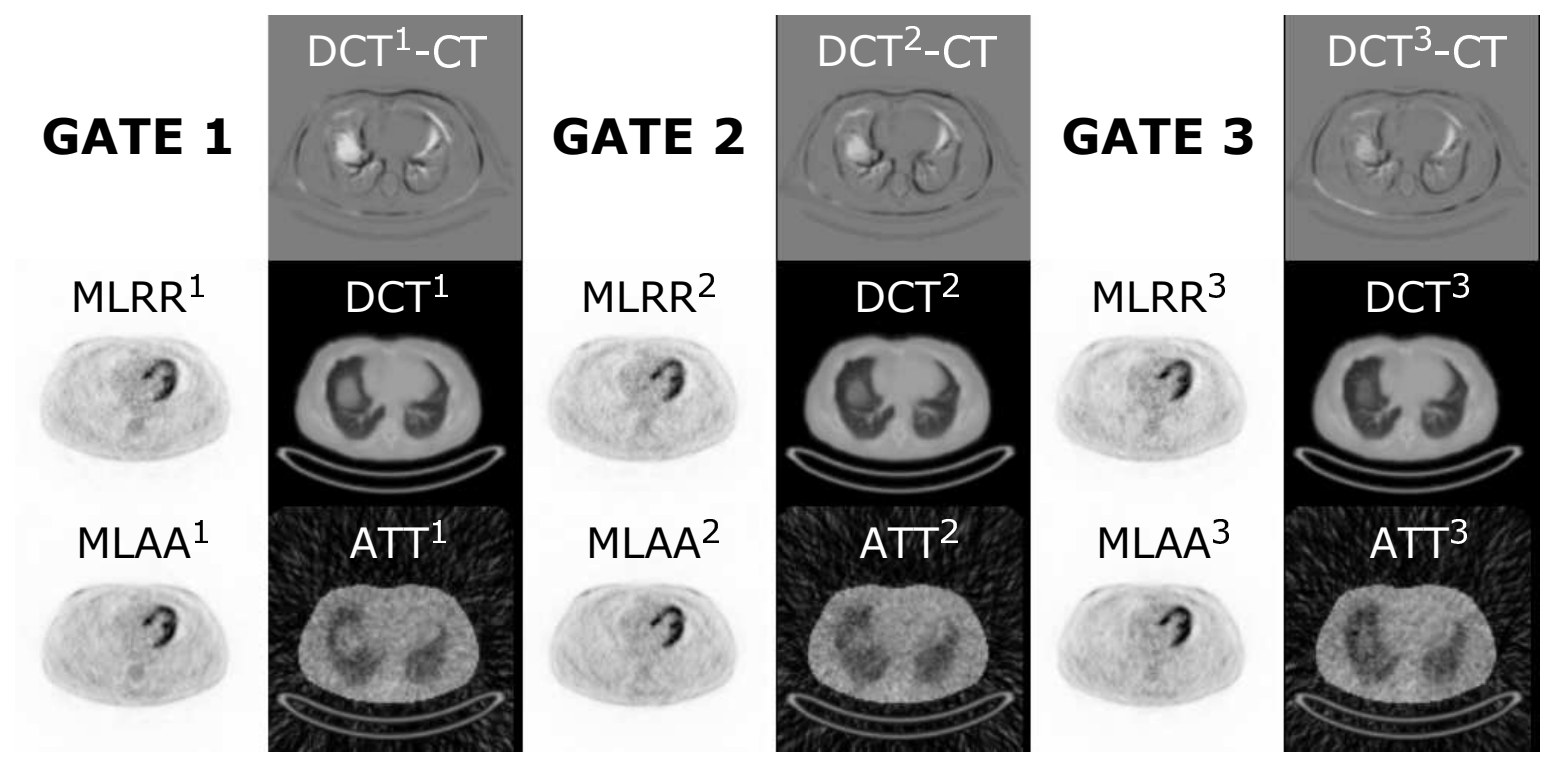

Figure 10: Results for the gated TOF-PET emission data. The superscripts denote the gate number. For each of the three gates, the following images are shown: MLRR activity and attenuation images (centre row), the MLAA activity and attenuation images (bottom row) and the difference between the MLRR attenuation image and the original CT-based attenuation (top right).

in case of between-scan and in-scan motion.

Although methods have been proposed that make use of the invaluable CT (Panin et al. 2012) or MR (Salomon et al. 2011; Li et al. 2013) data, the methods assume a perfect activity and attenuation alignment. In this contribution we use the available CTbased attenuation images and non-rigidly align them to the emission data. By doing so we obtain aligned attenuation images while approximately preserving the resolution and signal-to-noise ratio of the CT-images. Furthermore, since the CT-based attenuation images are pre-corrected for the appropriate photon energy of $511 \mathrm{keV}$, no extra handling of the data is required to correct for the unknown scale in the joint estimation problem (Defrise et al. 2012).

The proposed MLRR algorithm builds on the MLAA algorithm by replacing the update of the attenuation image by estimating transformation parameters that produce the required attenuation update. For each MLAA sub-iteration (with a 1:3 activity to attenuation update ratio), a total of 16 (back/)projections are required which only 2 are TOF (back/)projections. For reference, each MLEM sub-iteration involves only 2 TOF and 1 non-TOF (back/)projection operations. In addition to the same number of (back/)projections as MLAA, MLRR requires 3 incremental (rigid/nonrigid) motion estimations per iteration. In our implementation of the algorithm, each iteration of the MLRR algorithm roughly takes twice as long as an MLAA iteration.

The method was accelerated by using Nesterov's momentum which makes use of the previous attenuation update as well as the current update of MLTR. Our 
use of the momentum method is empirical, since it was used here to accelerate the MLTR algorithm, ignoring the fact that MLTR was interleaved with updates of the deformation. Nevertheless, it was found to yield a very significant acceleration. The effects of using Nesterov's momentum as well as the multi-resolution approach were investigated by means of the log-likelihood function and the RMSE of the MLRR attenuation reconstructions (figure 6). The convergence analysis showed a monotonic increase/decrease in the log-likelihood/RMSE values in the simulations. However, we should note that Nesterov's acceleration method is known to be non-monotonic. We anticipate that with a better TOF-resolution, a similar improvement would be expected for MLRR reconstructions as with MLAA reconstructions reported in (Rezaei et al. $2012 b)$.

In our experience and in the case of a non-rigid motion model, we were able to achieve stable results provided that the incremental displacement updates were small and locally smooth. The use of a multi-resolution scheme further improves the stability of the non-rigid registration. Although the proposed non-rigid update of (17) is count dependent, it reduces the influence of the vanishing attenuation updates in later iterations. We found the update of (17) to be better suited than the original regularised demons update with a fixed maximum displacement update in each iteration (Vercauteren et al. 2009). We do not expect problems due to the count-dependency of (17) in clinical practice as the strength of the quadratic regularisation $(\beta)$ of the estimated displacement field could be computed from the initial iterations to give the desired maximum update of the displacement fields.

In this study, the Gaussian fluid-like and diffusion-like smoothing parameters were chosen empirically; however, similar to (Yeo et al. 2008), a more extensive study is still required to optimise and to analyse the effect of the smoothing parameters on the estimated displacement field and on the quantitative accuracy of the reconstructed activity image. As illustrated in figure 4, noise on the emission data propagates into the MLRR attenuation map as uncertainties on the position of the contours in the deformed attenuation image. The results of the 2D noise analysis (figure 5) showed that noise propagation has no adverse effect on the noise in the reconstructed activity image. However, for some applications, e.g. when the deformed CT would also be used for defining regions of interest, it may be desirable that the deformation of the CT is as realistic as possible. For such applications a stronger constraining may be needed than the one we have applied in our experiments.

In the clinical patient scan, MLRR was able to produce an aligned attenuation image which effectively removed parts of the shadow-like artifacts observed in the MLEM activity reconstruction. The shadow-like artifacts which were observed above the dome of the liver were also absent in the MLRR activity reconstruction of the 3 respiratory gates; however, artifacts were still present near the lateral wall of the heart. We attribute this to the cardiac motion, present in each respiratory phase. MLRR produced a fairly large deformation near the lateral wall. We assume this deformation minimises the inconsistencies due to the cardiac motion. It is likely that these inconsistencies can 
not be eliminated entirely, since there is no stationary object that can explain the attenuation produced by an active and attenuating object which moved during the acquisition (Hamill et al. 2012; Rezaei et al. 2013b). Otherwise, the deformations produced by MLRR seem to be well in agreement with respiratory motion.

The registration step in the MLRR algorithm preserves the CT attenuation intensities, and is not mass preserving. The effects of utilising a mass preserving algorithm, and its comparison to the current intensity preserving algorithm remains to be studied. Furthermore, applying additional constraints on the estimated deformation field such as the ones which enforce local rigidity might provide a tool to better reflect internal motion. With the recent developments and interest in simultaneous PET/MR systems, adapting the method to also use the anatomical information gathered from the MR by combining MLRR (i.e. jointly estimating deformation and activity) with Salomon's approach (Salomon et al. 2011) (i.e. jointly estimating a small number of attenuation values and activity) could be fruitful.

\section{Conclusion}

The MLRR algorithm was proposed to make use of the high quality CT image and to iteratively reconstruct the activity image while deforming the CT-based attenuation image. Our 2D and 3D simulations indicate that the method is able to produce aligned activity and attenuation reconstructions similar to MLAA, with two advantages: the missing scale problem is solved automatically by using CT-based attenuation coefficients, and the resulting attenuation image is "almost free of noise". Furthermore, the improved alignment of CT and PET might also benefit the diagnostic value of the image pair, which remains to be investigated. However, some constraining of the estimated motion parameters is required to avoid inaccuracies observed mostly at high gradients of the attenuation image. The noise analysis showed that the corresponding activity reconstructions of MLRR were comparable to the ones produced by MLAA in terms of bias and variance. As expected, the activity reconstructions of the clinical scans produced by MLRR suffer less from motion-induced or mismatch artifacts than the reference MLEM activity reconstructions. In addition, the attenuation results of the gated patient data are in agreement with expected breathing motion.

\section{Acknowledgements}

This research is supported by a research grant (GOA) from KU Leuven and FWO project G027514N, and by Siemens Healthcare, Anderlecht, Belgium. The authors would like to thank A. Ribbens from KU Leuven for insightful discussions, J. Jones from Siemens Healthcare, Molecular Imaging for data processing and providing the data-based gating software. 


\section{Appendix A}

Given the time-of-flight PET emission data $y_{i t}$, the variables $\dot{L}$ and $\ddot{L}$ in $(5)$ can be computed from the Poisson log-likelihood (1) to give the MLTR attenuation update as:

$$
\begin{aligned}
\dot{L}_{j} & =\frac{\partial L}{\partial \mu_{j}}=\sum_{i t} l_{i j}\left(\bar{y}_{i t}-y_{i t}\right)\left(1-\frac{s_{i t}}{\bar{y}_{i t}}\right) \\
& =\sum_{i} l_{i j}\left(\left(\bar{y}_{i}-y_{i}\right)-\sum_{t}\left(\bar{y}_{i t}-y_{i t}\right) \frac{s_{i t}}{\bar{y}_{i t}}\right) \\
& \approx \sum_{i} l_{i j}\left(\bar{y}_{i}-y_{i}\right)\left(1-\frac{s_{i}}{\bar{y}_{i}}\right) \\
\ddot{L}_{j} & =\sum_{k} \frac{\partial^{2} L}{\partial \mu_{j} \partial \mu_{k}}=-\sum_{k} \sum_{i t} l_{i j} l_{i k}\left(\bar{y}_{i t}-s_{i t}\right)\left(1-\frac{y_{i t} s_{i t}}{\bar{y}_{i t}^{2}}\right) \\
& \approx-\sum_{i} l_{i j}\left(\left(\bar{y}_{i}-s_{i}\right)-\sum_{t}\left(\bar{y}_{i t}-s_{i t}\right) \frac{s_{i t}}{\bar{y}_{i t}}\right) \sum_{k} l_{i k} \\
& \approx-\sum_{i} l_{i j}\left(\bar{y}_{i}-s_{i}\right)\left(1-\frac{s_{i}}{\bar{y}_{i}}\right) \sum_{k} l_{i k}
\end{aligned}
$$

where, approximation (A.3) is achieved by assuming that the fraction $y_{i t} / \bar{y}_{i t} \approx 1$, and since it is imposed in computing $\ddot{L}$ (the denominator of the MLTR attenuation update) it only influences the convergence speed of the algorithm. Approximations (A.2) and (A.4) are achieved by assuming that the fraction $s_{i t} / \bar{y}_{i t}$ is roughly independent of the TOF-bin $t$, and can be replaced by the non-TOF fraction $s_{i} / \bar{y}_{i}$. This approximation eliminates the summation over the TOF index for both $\dot{L}$ and $\ddot{L}$. Since our approximation is exact for $s_{i t}=0$, we can expect it to be a good approximation for small $s_{i t}$. In cases where this is not so, the approximation does not affect the final solution (since in the noisefree case the problem of attenuation estimation has the same solution for non-TOF as well as for TOF emission data); however, it may adversely affect the noise propagation. The advantage of the approximation is that it reduces the computation time for the attenuation update.

Although the use of the TOF data has been suggested for the attenuation update in the joint estimation frame-work (Ahn et al. 2012), in our experiments the TOF information was ignored to reduce the computation time. The combination of (A.2) and (A.4) which we used, is the standard non-TOF MLTR update with an additive contribution (Van Slambrouck et al. 2014).

\section{Appendix B}

In this section, with some approximations we derive the displacement update (17) justifying the use of the demons algorithm (Thirion 1998) to update the deformation field. We parameterise the transformation by a displacement field, i.e. the deformation parameters $\Theta$ now consist of a vector $\boldsymbol{D}_{j}=\left(D_{j}^{x}, D_{j}^{y}, D_{j}^{z}\right)$ for every voxel $j$, and focus on 
the estimation of the displacement field that non-rigidly deforms $\mu^{\dagger}$ to an attenuation image that agrees best with the TOF-PET emission data according to the Poisson likelihood. The derivative in (9), with respect to an incremental displacement field $\boldsymbol{d}$ at voxel $j$, can now be approximated by:

$$
\frac{\partial \delta \mu_{k}[\boldsymbol{d}]}{\partial \boldsymbol{d}_{j}}=\frac{\partial \mu_{k}\left[\boldsymbol{D}^{(n)}+\boldsymbol{d}\right]}{\partial \boldsymbol{d}_{j}} \approx\left(\boldsymbol{\nabla} \mu\left[\boldsymbol{D}^{(n)}\right]\right)_{j} \delta_{j k}
$$

where $\left(\boldsymbol{\nabla} \mu\left[\boldsymbol{D}^{(n)}\right]\right)_{j}$ is the gradient of the deformed attenuation image at voxel $j$ with respect to the image coordinates $(x, y, z)$, and $\delta_{j k}$ is the Kronecker delta. This approximation assumes that the value in a pixel is independent of the deformation in other pixels. In reality however, there will be some dependence on neighbouring voxels due to unavoidable interpolations.

Furthermore, using the first order Taylor series expansion of $\mu_{j}\left[\boldsymbol{D}^{(n)}+\boldsymbol{d}\right]$ together with (B.1) we find $\|$ :

$$
\delta \mu_{j}[\boldsymbol{d}] \approx\left(\boldsymbol{\nabla} \mu\left[\boldsymbol{D}^{(n)}\right]\right)_{j} \boldsymbol{d}_{j}
$$

Inserting (B.1) and (B.2) in (9) we obtain:

$$
\frac{\partial \tilde{S}}{\partial \boldsymbol{d}_{j}}=\left(\dot{L}_{j}^{(n)}+\ddot{L}_{j}^{(n)}\left(\boldsymbol{\nabla} \mu\left[\boldsymbol{D}^{(n)}\right]\right)_{j} \boldsymbol{d}_{j}\right)\left(\boldsymbol{\nabla} \mu\left[\boldsymbol{D}^{(n)}\right]\right)_{j}=0
$$

The solution of (B.3) is not unique. By taking the minimum norm solution (the same choice was made in (Thirion 1998; Vercauteren et al. 2009)) an incremental displacement $\boldsymbol{d}_{j}$ is estimated in the direction of $\left(\boldsymbol{\nabla} \mu\left[\boldsymbol{D}^{(n)}\right]\right)_{j}$, which reduces the problem to a set of 1D problems with solution

$$
\boldsymbol{d}_{j}^{(n)}=-\frac{\left(\boldsymbol{\nabla} \mu\left[\boldsymbol{D}^{(n)}\right]\right)_{j}}{\left\|\left(\boldsymbol{\nabla} \mu\left[\boldsymbol{D}^{(n)}\right]\right)_{j}\right\|^{2}} \frac{\dot{L}_{j}^{(n)}}{\ddot{L}_{j}^{(n)}}=\frac{\left(\boldsymbol{\nabla} \mu\left[\boldsymbol{D}^{(n)}\right]\right)_{j}}{\left\|\left(\boldsymbol{\nabla} \mu\left[\boldsymbol{D}^{(n)}\right]\right)_{j}\right\|^{2}} \delta \mu_{j}^{(n)}
$$

where $\delta \mu^{(n)}$ is the attenuation update of MLTR (5). Equation (B.4) is the unconstrained optical flow equation commonly used in image registration methods.

Since the registration problem is highly under-determined, it is often necessary to regularise the estimated displacement fields. This is typically achieved by adding a penalty term that favours smaller values of displacements $\boldsymbol{d}_{j}$. Adding a quadratic penalty term to the surrogate function (9), we have:

$$
\tilde{S}_{r e g}(\mu[\boldsymbol{D}+\boldsymbol{d}], y)=\tilde{S}(\mu[\boldsymbol{D}+\boldsymbol{d}], y)-\sum_{j} \frac{1}{2} \beta\left\|\boldsymbol{d}_{j}\right\|^{2}
$$

where $\beta$ determines the strength of the penalty term. With the same approximations, the incremental displacement update is then given in (17), where $\beta / \ddot{L}_{j}^{(n)}$ helps to stabilise the incremental displacement estimate $\boldsymbol{d}^{(n)}$ at low gradient values of the deformed attenuation image.

\| In this context all vector multiplications are inner products. 


\section{References}

Ahn, S., H. Qian, and R. M. Manjeshwar (2012). "Convergent iterative algorithms for joint reconstruction of activity and attenuation from time-of-flight PET data". In: 2012 IEEE Nucl. Sci. Symp. Med. Imaging Conf. Rec. IEEE, pp. 3695-3700.

Alessio, A. M. et al. (2010). "Attenuation-emission alignment in cardiac PET/CT based on consistency conditions". In: Med. Phys. 37.3, p. 1191.

Boellaard, R. et al. (2014). "Accurate PET/MR quantification using time of flight MLAA image reconstruction". In: Mol. Imaging Biol. 16.4, pp. 469-477.

Bromiley, A. et al. (2001). "Attenuation correction in PET using consistency conditions and a three-dimensional template". In: IEEE Trans. Nucl. Sci. 48.4, pp. 1371-1377.

Bronnikov, A. V. (2000). "Reconstruction of attenuation map using discrete consistency conditions." In: IEEE Trans. Med. Imaging 19.5, pp. 451-62.

Cachier, P. and N. Ayache (2004). "Isotropic Energies, Filters and Splines for Vector Field Regularization". In: J. Math. Imaging Vis. 20.3, pp. 251-265.

Conti, M. (2011). "Why is TOF PET reconstruction a more robust method in the presence of inconsistent data?" In: Phys. Med. Biol. 56.1, pp. 155-68.

Defrise, M. et al. (2008). "Continuous and discrete data rebinning in time-of-flight PET." In: IEEE Trans. Med. Imaging 27.9, pp. 1310-22.

Defrise, M., A. Rezaei, and J. Nuyts (2012). "Time-of-flight PET data determine the attenuation sinogram up to a constant." In: Phys. Med. Biol. 57.4, pp. 885-99.

- (2014). "Transmission-less attenuation correction in time-of-flight PET: analysis of a discrete iterative algorithm." In: Phys. Med. Biol. 59.4, pp. 1073-95.

Hamill, J. J. and V. Y. Panin (2012). "TOF-MLAA for attenuation correction in thoracic PET/CT". In: 2012 IEEE Nucl. Sci. Symp. Med. Imaging Conf. Rec. IEEE, pp. 4040-4047.

Jakoby, B. W. et al. (2011). "Physical and clinical performance of the mCT time-of-flight PET/CT scanner." In: Phys. Med. Biol. 56.8, pp. 2375-89.

Joseph, P. M. (1982). "An Improved Algorithm for Reprojecting Rays through Pixel Images." In: IEEE Trans. Med. Imaging 1.3, pp. 192-196.

Kim, D., S. Ramani, and J. A. Fessler (2014). "Combining Ordered Subsets and Momentum for Accelerated X-ray CT Image Reconstruction." In: IEEE Trans. Med. Imaging 0062.c, pp. 1-12.

Kinahan, P. E., B. H. Hasegawa, and T. Beyer (2003). "X-ray-based attenuation correction for positron emission tomography/computed tomography scanners." In: Semin. Nucl. Med. 33.3, pp. 166-79.

Kudo, H. and H. Nakamura (2001). "A new approach to SPECT attenuation correction without transmission measurements". In: 2000 IEEE Nucl. Sci. Symp. Conf. Rec. Vol. 2. 2. IEEE, pp. 13/58-13/62.

Lange, K. and J. A. Fessler (1995). "Globally convergent algorithms for maximum a posteriori transmission tomography." In: IEEE Trans. Image Process. 4.10, pp. $1430-8$. 
Li, H., G. El Fakhri, and Q. Li (2013). "Direct MAP Estimation of Attenuation Sinogram using TOF PET Data and Anatomical Information". In: 2013 Fully Three-Dimensional Image Reconstr. Radiol. Nucl. Med. Proc. Vol. 3, pp. 405-408.

Mehranian, A. and H. Zaidi (2015a). "Impact of Time-of-Flight PET on Quantification Errors in MR Imaging-Based Attenuation Correction". In: J. Nucl. Med. 56, pp. 635-641.

- (2015b). "Joint estimation of activity and attenuation in whole-body TOF PET/MRI using constrained Gaussian mixture models". In: IEEE Trans. Med. Imaging 0062.i, pp. 1-1.

Natterer, F. (1993). "Determination of tissue attenuation in emission tomography of optically dense media". In: Inverse Probl. 9.6, pp. 731-736.

Natterer, F. and H. Herzog (1992). "Attenuation correction in positron emission tomography". In: Math. Methods Appl. Sci. 15.5, pp. 321-330.

Nesterov, Y. (1983). "A method of solving a convex programming problem with convergence rate O (1/k2)." In: Sov. Math. Dokl. 27.2.

Panin, V. Y. et al. (2004). "Application of discrete data consistency conditions for selecting regularization parameters in PET attenuation map reconstruction". In: Phys. Med. Biol. 49.11, pp. 2425-2436.

Panin, V. Y. et al. (2012). "Reconstruction of uniform sensitivity emission image with partially known axial attenuation information in PET-CT scanners". In: 2012 IEEE Nucl. Sci. Symp. Med. Imaging Conf. Rec. (2012 NSS/MIC). IEEE, pp. 2166-2173.

Rezaei, A., J. Nuyts, and M. Defrise (2012a). "Analytic reconstruction of the attenuation from 3D time-of-flight PET data". In: 2012 IEEE Nucl. Sci. Symp. Med. Imaging Conf. Rec. (2012 NSS/MIC). IEEE, pp. 2330-2333.

Rezaei, A. et al. (2012b). "Simultaneous reconstruction of activity and attenuation in time-of-flight PET." In: IEEE Trans. Med. Imaging 31.12, pp. 2224-33.

Rezaei, A. and J. Nuyts (2013a). "Simultaneous reconstruction of the activity image and registration of the CT image in TOF-PET". In: 2013 IEEE Nucl. Sci. Symp. Med. Imaging Conf. Rec. (2013 NSS/MIC). IEEE, pp. 1-3.

Rezaei, A., J. Nuyts, and M. Defrise (2013b). "The Effect of Motion on Joint Estimates of Activity and Attenuation from Time-of-Flight PET Data". In: 2013 Fully ThreeDimensional Image Reconstr. Radiol. Nucl. Med. Proc. 2, pp. 297-300.

Rezaei, A., M. Defrise, and J. Nuyts (2014). "ML-reconstruction for TOF-PET with simultaneous estimation of the attenuation factors." In: IEEE Trans. Med. Imaging 33.7, pp. 1563-72.

Salomon, A. et al. (2011). "Simultaneous reconstruction of activity and attenuation for PET/MR." In: IEEE Trans. Med. Imaging 30.3, pp. 804-13.

Segars, W. P. et al. (2010). "4D XCAT phantom for multimodality imaging research". In: Med. Phys. 37.9, p. 4902.

Thirion, J. P. (1998). "Image matching as a diffusion process: an analogy with Maxwell's demons". In: Med. Image Anal. 2.3, pp. 243-260. 
van Elmpt, W. et al. (2011). "Optimal gating compared to 3D and 4D PET reconstruction for characterization of lung tumours." In: Eur. J. Nucl. Med. Mol. Imaging 38.5, pp. 843-55.

Van Slambrouck, K. and J. Nuyts (2014). "Reconstruction Scheme for Accelerated Maximum Likelihood Reconstruction: The Patchwork Structure". In: IEEE Trans. Nucl. Sci. 61.1, pp. 173-181.

Vercauteren, T. et al. (2009). "Diffeomorphic demons: efficient non-parametric image registration." In: Neuroimage 45.1 Suppl, S61-72.

Wang, G. and J. Qi (2009). "Generalized algorithms for direct reconstruction of parametric images from dynamic PET data." In: IEEE Trans. Med. Imaging 28.11, pp. 1717-26.

Watson, C. (2007). "Extension of Single Scatter Simulation to Scatter Correction of Time-of-Flight PET". In: IEEE Trans. Nucl. Sci. 54.5, pp. 1679-1686.

Welch, A. et al. (1998). "Attenuation correction in PET using consistency information". In: IEEE Trans. Nucl. Sci. 45.6, pp. 3134-3141.

Yeo, B. T. T. et al. (2008). "Effects of registration regularization and atlas sharpness on segmentation accuracy". In: Med. Image Anal. 12.5, pp. 603-615. 